\begin{tabular}{ccc} 
Available online at www.sinjas.journals.ekb.eg & $\overline{\text { ARU-EGYPT }}$ \\
\hline SCREENED BY SINAI Journal of Applied Sciences
\end{tabular}

\title{
IMPACT OF BANKING SECTOR DEVELOPMENT ON ECONOMIC GROWTH IN MALAYZIA
}

\author{
Samaa I.S. Ahmed ${ }^{1^{*}}$, M.E. Hassoona ${ }^{2}$ and M.R. Ismaiel ${ }^{3}$ \\ 1. Soc. Sci. Studies and Res. Dept., Asian Studies and Res. Inst., Zagazig Univ., Egypt \\ 2. Dept. Econ., Fac. Commerce, Azhar Univ., Egypt \\ 3. Dept. Agric. Econ., Fac. Agric., Zagazig Univ., Egypt
}

\begin{tabular}{l} 
ARTICLE INFO \\
\hline Article history: \\
Received: $14 / 03 / 2021$ \\
Revised: $09 / 04 / 2021$ \\
Accepted: $21 / 04 / 2021$ \\
Available online: $29 / 04 / 2021$ \\
\hline Keywords: \\
Banking sector, \\
privatization, \\
economic growth, \\
Malayzia, \\
albomutera Bank \\
Check for \\
updates
\end{tabular}

\begin{abstract}
This research examines the experience of Malayzia, which was able to form strong banking entities after the severe economic crisis it was exposed to in 1997. Malayzia is one of the fastest countries to emerge from the Asian crisis. The importance of the research is represented in reviewing the country's banking experiences and the impact of reforms on economic growth in Malayzia during the period from (1980-2014). This research aims to identify the major developments that have taken place in the banking sector and to understand their results. Among the most important results of the research is a study of reforming the Malayzian banking sector, and the application of the privatization policy. Malayzia has achieved diversification in the traditional banking sector and the Islamic banking system, which coexist and work together. Among the most important indicators of the Malayzian banking sector, according to the balance sheets during the period (2007-2014), is the risk-weighted capital adequacy ratio, which reached an average of $10.9 \%$ and the basic capital adequacy ratio, which reached an average of $9.33 \%$. Also, the return on assets ratio reached an average of $1.48 \%$. Among the most important recommendations reached by the research is the necessity to adhere to structural reform programs and link them with economic, financial and legislative reform, work to merge small banks, increase bank branches and provide medium and long-term loans to create investment opportunities and obtain an appropriate profit margin.
\end{abstract}

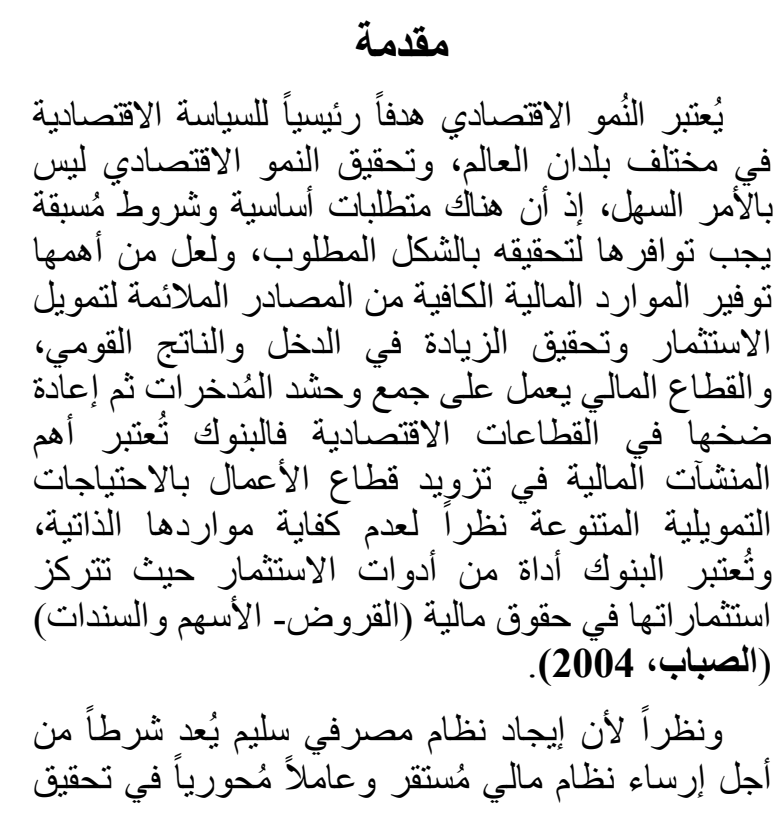

نمو اقتصادي وتتمية مستدامة فمن الصعوبة بمكان أن

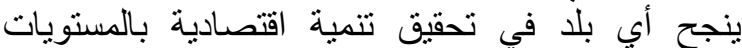

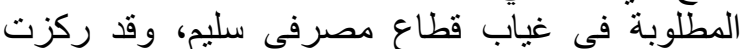

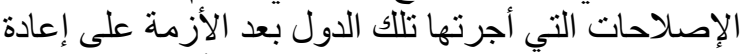

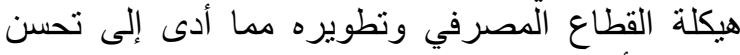
كبير في أدائه (علي، 2007).

ولقد تَم تناول القطاع المَصرفي الماليزي، لأن ماليزيا

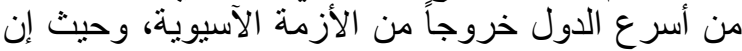

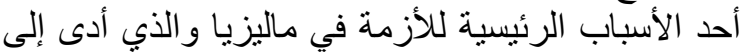

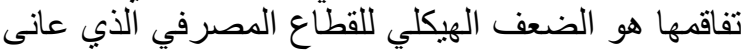

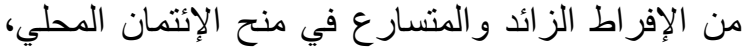

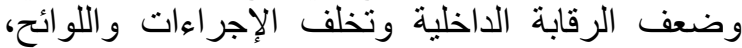

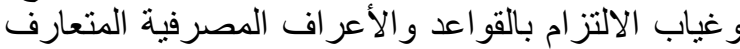

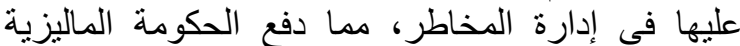

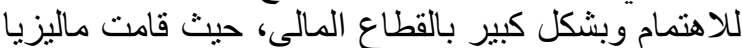

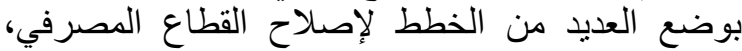

* Corresponding author: E-mail address: samaaibrahem7@gmail.com https://doi.org/ 10.21608/SINJAS.2021.67821.1011

(C) 2020 SINAI Journal of Applied Sciences. Published by Fac. Environ. Agric. Sci., Arish Univ. All rights reserved. 


$$
\begin{aligned}
& \text { مَنهج البحث } \\
& \text { ينقسم منهج البحث إلى جُزئين: } \\
& \text { الجُزء النظري }
\end{aligned}
$$

يتم استعراض الإصلاحات التي تمت في ماليزيا

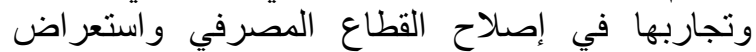

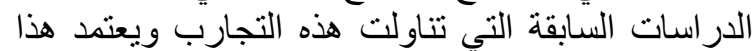

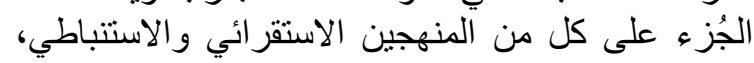

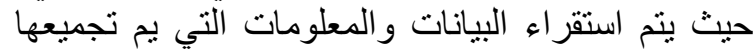

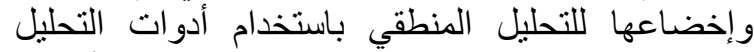

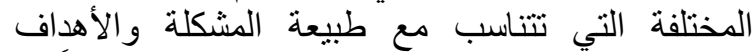

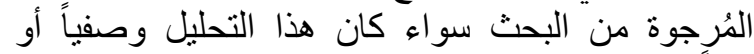
كمباً، وذلك بغرض استنباط الحقائق المرتبطة بالمشكلة المباء

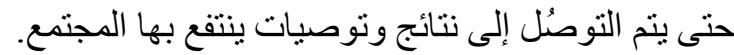

$$
\text { الار اسة القياسية }
$$

تعتمد الدراسة على استخدام نموذج الانحدار الذاتي

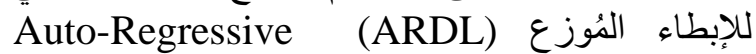

Chung المقدم بواسطة Distributed Lag-model وباستعمال المتغيرات التالية: معدل نمو

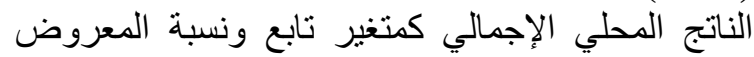

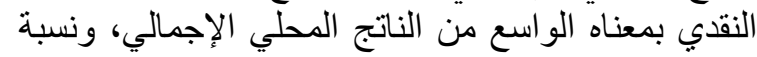

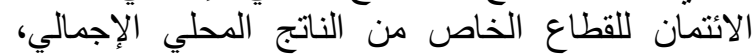

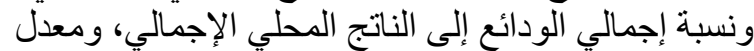
التضخم وسعر الصرف الحقيقي كمتغير التي مستقلة.

\section{النُمو الاقتصادي}

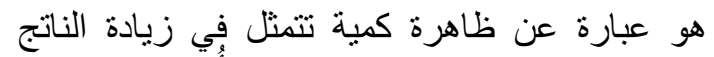

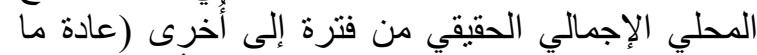
تكون سنة) وزيادة نصيب الفرد منهاه. ولم تُحقق ماليزيا

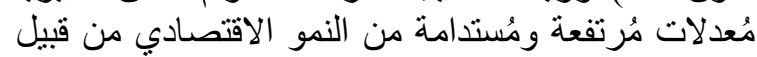

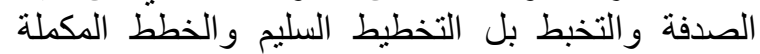

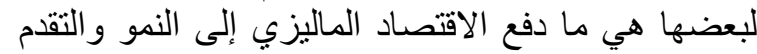

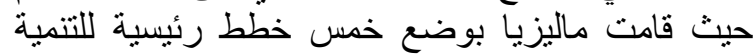

وهي (Abdul Kareem, 1988):

ـ السياسة الاقتصادية الجديدة 1971-1990 وكانت تهدف إلى تخفيض نسبة الفقر للوصول إلى اللى القضاء علئ عليه نهائياً

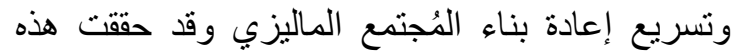
الخطة الكثير من أهدافها.

ـ سياسة التتمية الوطنية 1991م -2000م وقد سعت هذه التهي السياسة إلى تحقيق التوازن بين التنمية الاقتصادية وبين

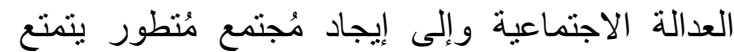

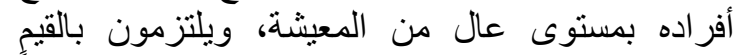

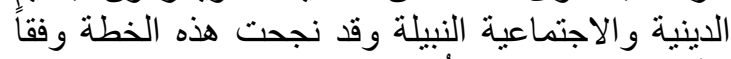
للأهداف المرجو تحقيقُها منها. - سياسة الرؤية الوطنية (2001 - 2010) أعطَت اهنماماً أكبر للتعامل مع العولمة.
وتكوين كيانات مصرفية محلية قوية تتكامل مع بعضها

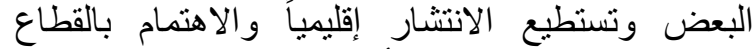

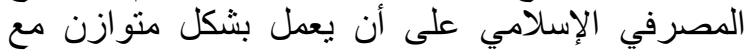

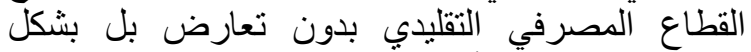

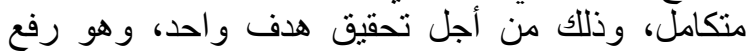

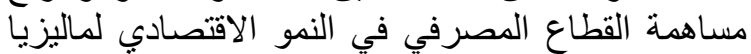

(اندراوس، 2008).

مُشكلة البحث

تتمثل المشكلة في الإجابة عن التساؤلات التالية: ـ ماهي سياسات الإصلاح و التطوير التي تمت في ماليزيا بعد الأزمة الاقتصادية التي لحقت بشرق آليّات 1997

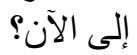

ـ كيف ساهمت سياسات الإصلاح في الرفع من كانياء كفاءة الأداء في القطاع المصرفي في دولة ماليزيا ؟

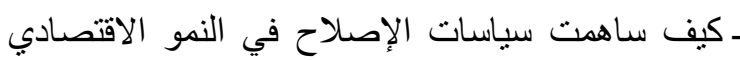

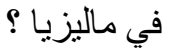
أهمية البحث ماليزاب

ـ استعر اض تجارب دولة استطاعت تكوين كيانات مصرفية قوية بعد أزمة اقتصادية طاحنة تعرضت لهانيات لهان عام 1997م وحققت معدلات نمو مرتفعة. ـ تقديم لمحة عن الإصلاحات المصرفية الأخيرة التي أُجريت في ماليزيا وأثر ذلاتل على نطور القطاع

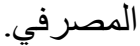

ـ تأثير هذه الإصلاحات على النمو الاقتصادي في ماليزيا خلال الفترة من عام (1980-2014) وبالألتالي إمكانية الإستفادة من هذه التجارب وتطبيقامها في مصر.

\section{هدف البحث}

يهدف هذا البحث إلى التعرُف على التطور ات الكبيرة

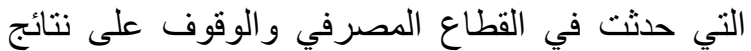

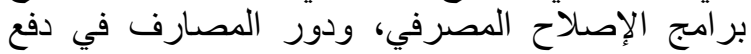

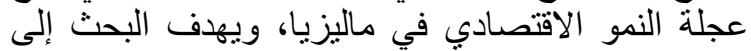

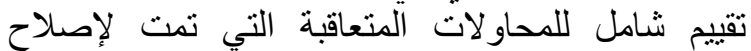

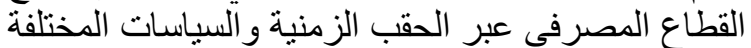

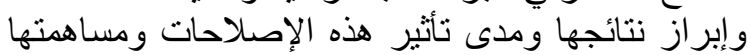
في النمو الاقتصادي لماليزيا.

$$
\text { فرضية البحث }
$$

يرتكز على فرضين أساسيين مفادهما:

1- توجد علاقة بين تحسين المؤشرات المصرفية العزئ (السيولة

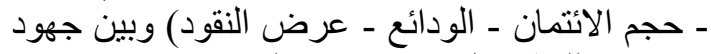

الإصلاح للقطاع المصرفي في ماليزيا.

2-توجد علاقة بين تطور القطاع المصرفي والنمو مائيا. الاقتصادي في ماليزيا. 


\section{القطاع المصر في بعد الأزمة}

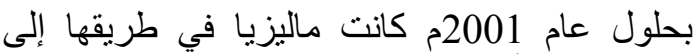

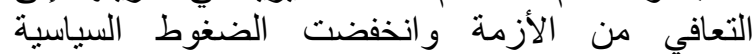

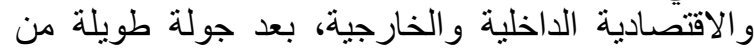

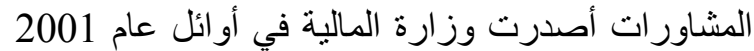

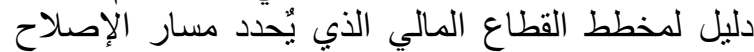

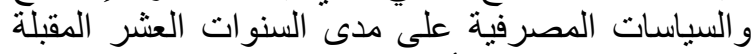

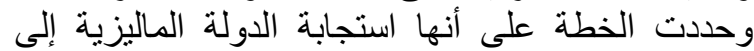

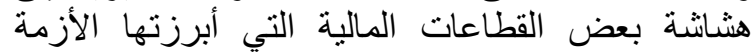

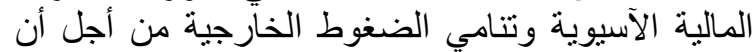

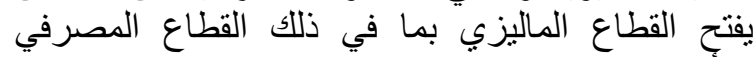

(الأنصاري، 1995).

بعد ذللك تم وضع رؤية للقطاع المصرفي الماليزي في

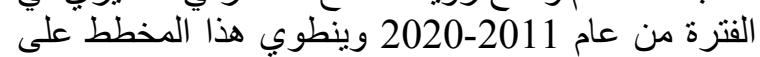

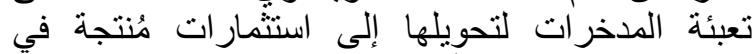

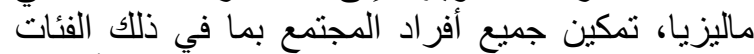

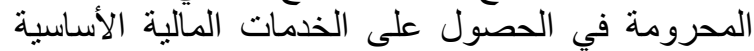

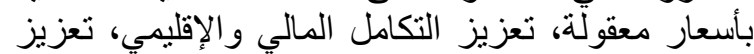
النظام المالي الإسلامي.

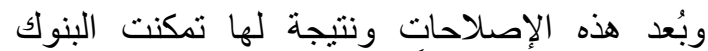

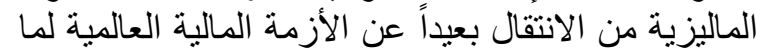

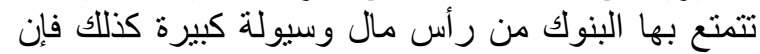

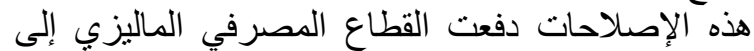

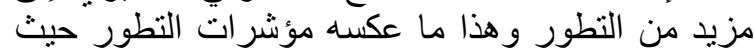

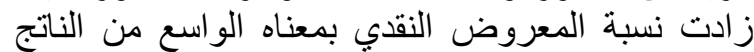

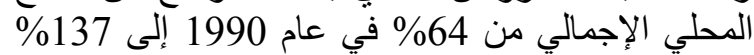

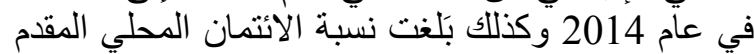

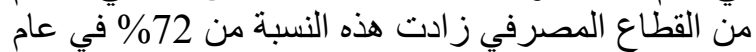

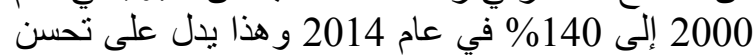
مؤشرات التطور مع جهود الإصلاح المبذولة (شحاته،

.1999

\section{مؤشرات القطاع المصرفي الماليزي وفقاً للميزانيات} العمومية خلال الفترة من (2007-2014)

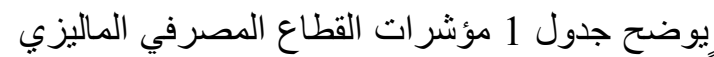
وفقاً للميزانيات العمومية خلال الفئرات الفترة من (20072014) ومنه يتبين أن:

نسبة كفاية رأس المال المرجح بالمخاطر (2014) ومنه بترن

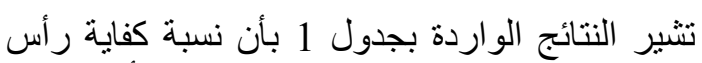

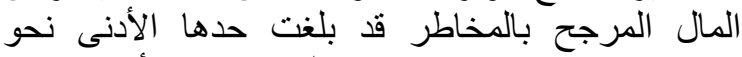

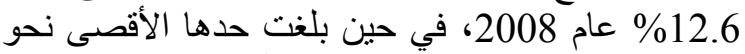

15.8 \% عام 2009، بمتّوسط عام بلغ نحو 10.9 \%

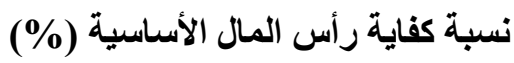

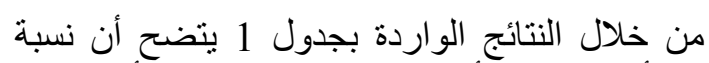

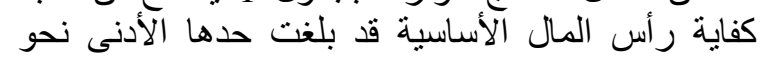

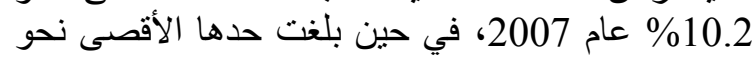

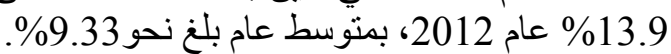

ـ النموذج الاقتصادي الجديد (2011-2020) وهى ثُركز

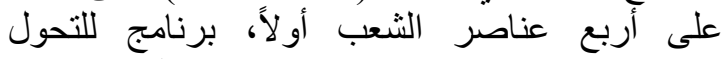
الاقتصادي وتحديد نتائج مُحددة تسعى إلى تحقي تحقيقها، خطة ماليزيا العاثرة (2011 -2015).

- رُؤية (2020) نرتكز على أربع عناصر وهى القئلئ القومية

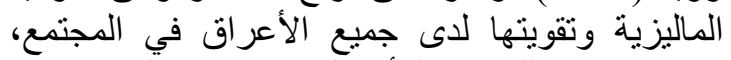

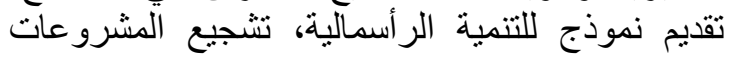
الخاصة، الاهتمام بدور الإسلام. للاندة الرالية

\section{إصلاحات القطاع المصرفي الماليزي قبل وبعد الأزمة}

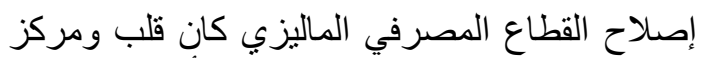
السياسة الاقتصادية الجديدة لَماليزيا وقالِ بدأ هذا الإصلاح

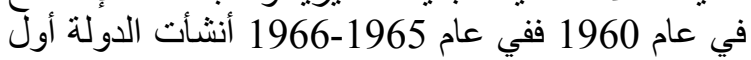

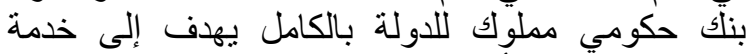

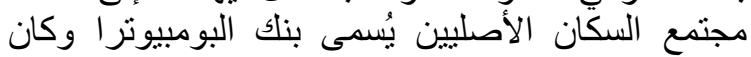

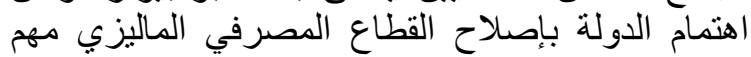

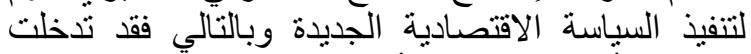

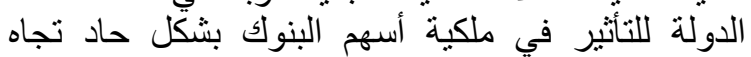

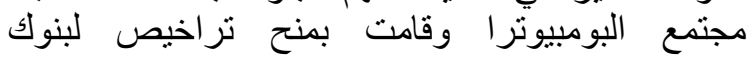

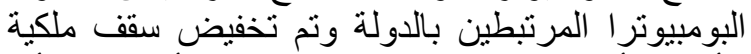

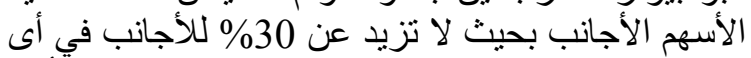

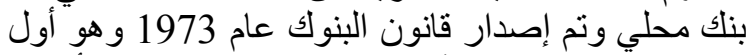

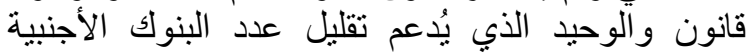

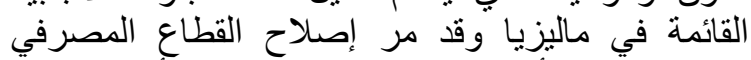
الماليزي قبل الأزمة بعدة مر احل المرحلة الآلئل

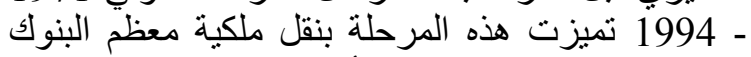

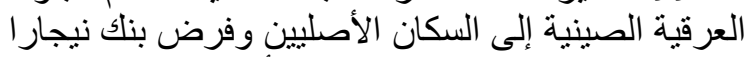

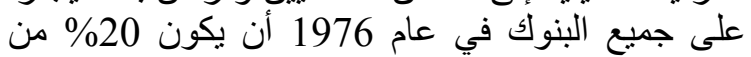

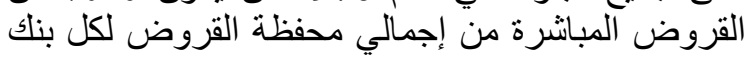

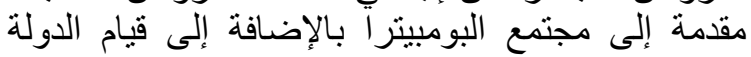

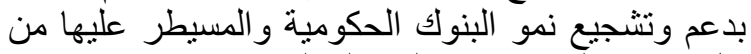

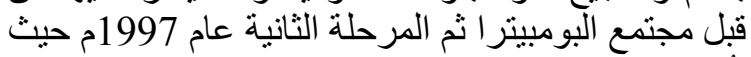
عُرفت هذه المرحلة بسيادة الخطة الاقتصنادية الجديدة

.(Terrick and Kindlebebarger, 1985)

\section{نقاط الضعف في القطاع المصرفي الماليزي}

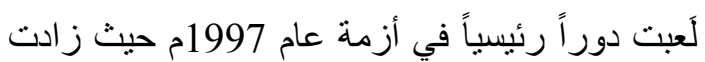
من تعرض المؤسسات المالية لمجموعة أزماتة من التهايدات

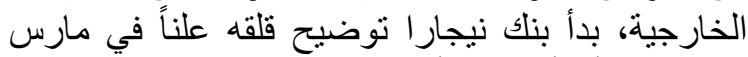

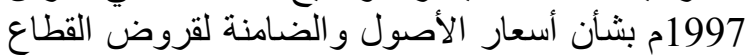

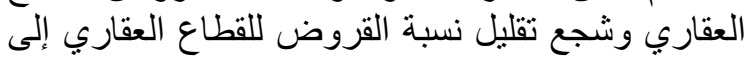

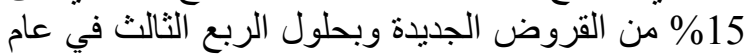

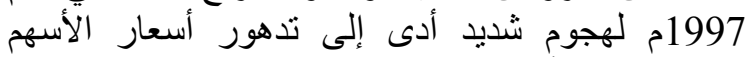

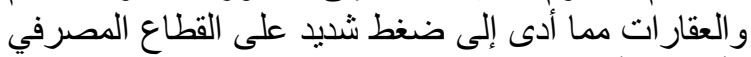

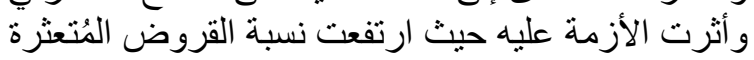

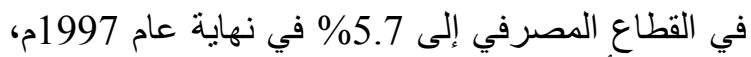

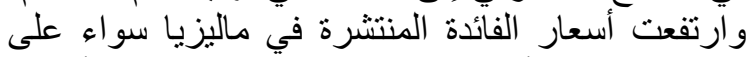

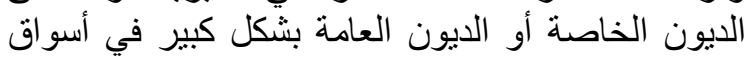
الائتمان الدولة لانكماش التصادها (الجنابي، 2009). 
جدول 1. مؤشرات القطاع المصرفي الماليزي وفقاً للميزانيات العمومية خلال الفترة من (2014-2007)

\begin{tabular}{|c|c|c|c|c|c|c|c|}
\hline المتروضي & 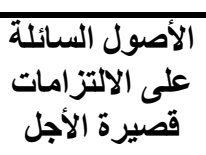 & الأصول السائلة & نلية العلى حقوق & نسبة الأصولئ & الأسباسية كفاية المالة & نبالمة كفاية رأس المرج & البيان \\
\hline 3.2 & 38.5 & 14.3 & 19.8 & 1.50 & 10.2 & 13.2 & 2007 \\
\hline 2.2 & 41.9 & 14.6 & 18.6 & 1.50 & 10.6 & 12.6 & 2008 \\
\hline 10.8 & 42.9 & 14.20 & 14 & 1.20 & 13.8 & 15.8 & 2009 \\
\hline 2.3 & 48.10 & 15.60 & 16.6 & 1.50 & 13. & 14.8 & 2010 \\
\hline 1.8 & 45.50 & 16 & 17.4 & 1.60 & 13.2 & 15.1 & 2011 \\
\hline 1.4 & 42.5 & 13.8 & 17.4 & 1.60 & 13.9 & 15.7 & 2012 \\
\hline 1.3 & 36.5 & 11.6 & 15.9 & 1.50 & 0 & 0 & 2013 \\
\hline 1.2 & 42.6 & 13.14 & 15.2 & 1.50 & 0 & 0 & 2014 \\
\hline 3.02 & 42.31 & 14.15 & 16.86 & 1.48 & 9.33 & 10.9 & المتوسط \\
\hline
\end{tabular}

2014، في حين بلغت حدها الأقصى حوالي 1009 عام بلي 2009، بمتوسط عام بلغ حو الي 3.02،

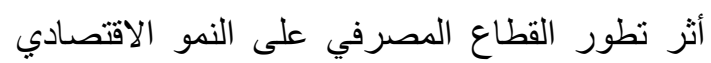

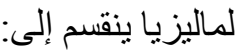

\section{الأساليب القياسية المستخدمة}

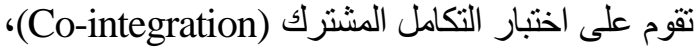

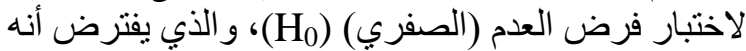

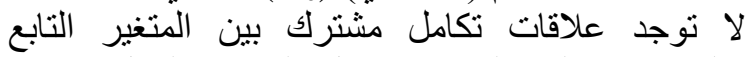

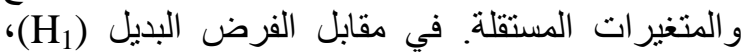

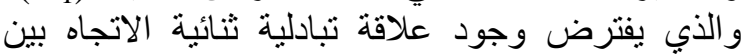

المتغير التابع والمتغير وجرد علاتة المستقلة (Bi-Directional).

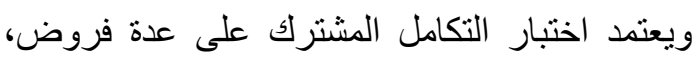
وتتمثل هذه الفروض في الآتي:

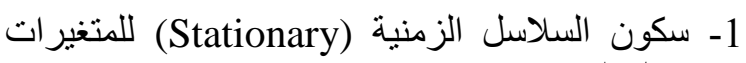

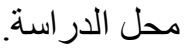
2- وجود علاقة توازنية بين المتغيرين في الأجل الطويل.

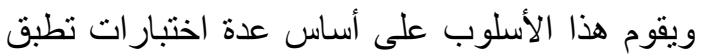
على السلاسل الزمنية محل الدر اسة، تتمثل في الآتي: 1- فحص سكون بيانات السلاسل الزمنية (Stationary)؛

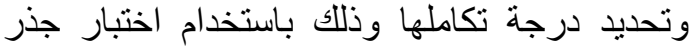

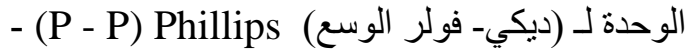
Augmented Dicky -Fuller (ADF)،Perron

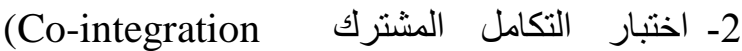
(Analysis)

\section{نسبة العائد على الأصول (\%)} تشير النتائج الواردة بجدول 1 بأن نسبة العائد على الأى

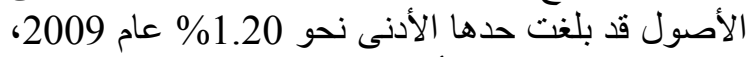

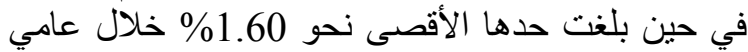

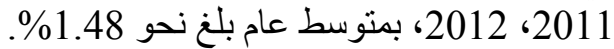

\section{نسبة العائد على حقوق الملكية (\%)}

من خلال النتائج الواردة بجدول 1 يتضح أن نسبة

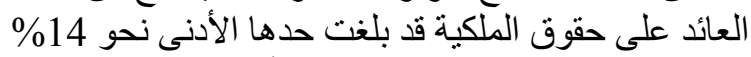

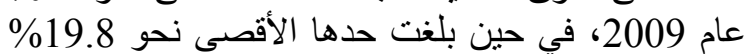

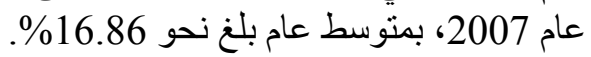
الأصول السائلة على إجمالي الأصول:

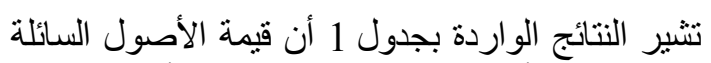

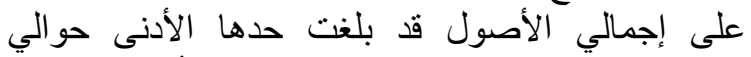
11.6 عام 2013، في حين بلغت حدها الأفي الأقصى حوالي

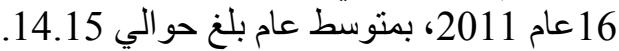

الأصول السائلة على الالتزامات قصيرة الأجل

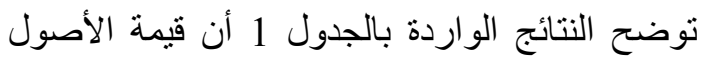

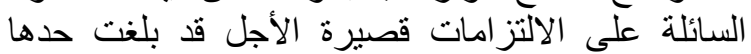
الأدنى حوالي 36.5عام 2013، الألئرات في حين بلغت حدها الأقصى حوالي 45.50عام 2011، بمتوسط عام بلغ بلغ حوالي 42.31. صافي القروض المُتعثرة

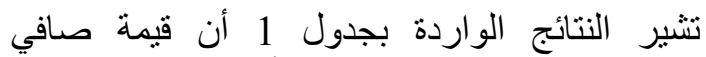
القروض المتعثرة قد بلغت حدها الأدنى حوالي 1.2 عام 
5- حققت ماليزيا التنوع في القطاع المصرفي حيث

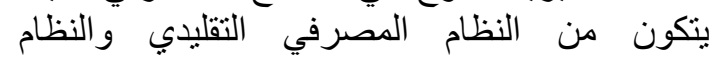

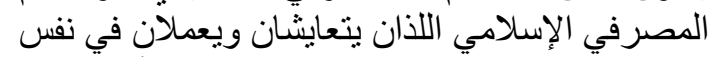
الوقت و هذه الاستر اتيجية تتناسب مع حقيقة أن ماليزيان

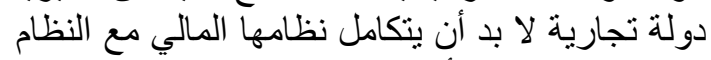

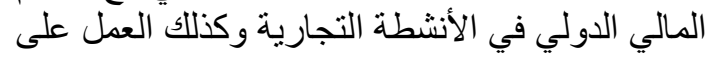

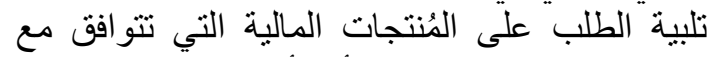

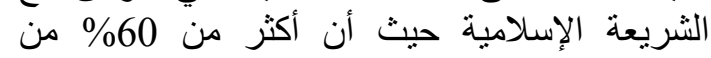
سكانها مسلمين.

6- القطاع المصرفي الماليزي لعب دوراً رئيسياً في في فئي

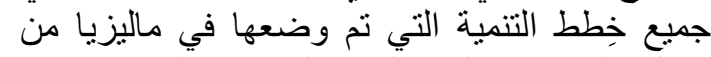

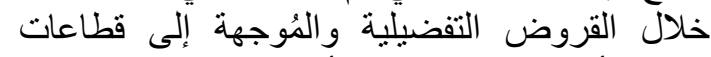
بعينها أو إلى السكان الأصليين لاعم تجارتهم وصناعنهم.

7- قامت الدولة الماليزية بالعمل بجد للحد من تعرض الإلما

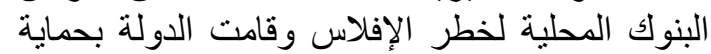

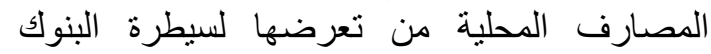

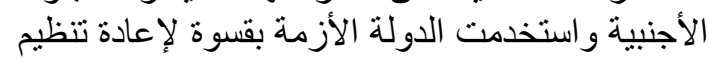
القطاع المصرفي من خلال الإندماجات على الإعلى الصعيد المحلي.

8- قامت الدولة الماليزية بالعمل على دمج المصارف

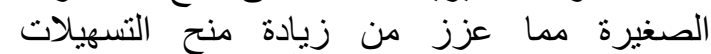

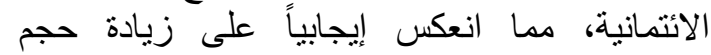
التسهيلات الممنوحة للاقتصاد وبالتاليالي دفع عجلة ائلة

النمو في ماليزيا.

التوصيات

1 - ضرورة الالتزام بير امج الإصلاح الهيكلي وربطها مع الإحساح

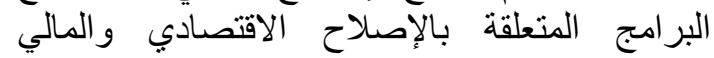

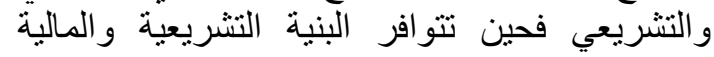

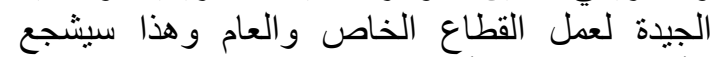
الأفر اد على إيداع أمو الهم لدى المصارف العارف.

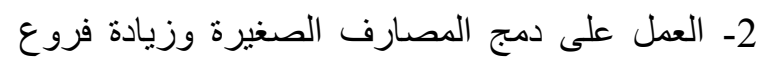
المصارف في المناطق الريقارفية سيعزز من إمكانية زيادة منح التسهيلات الائتمانية، مما سينية سئعكس إيجابياً

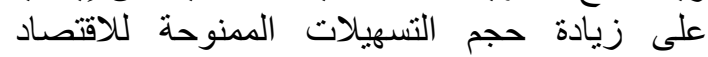
وبالتالي دفع عجلة النمّو في الدولة.

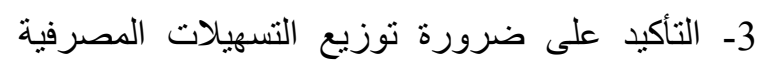

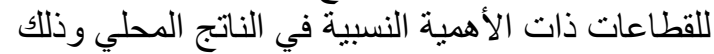

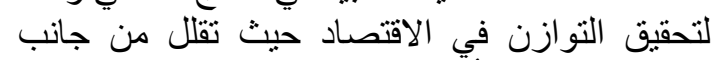
القطاعات التي لانُساهم في الاقتصـاد.

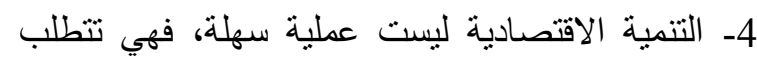

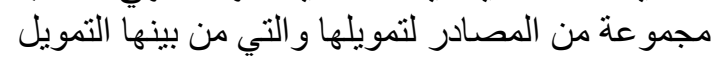

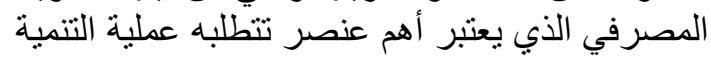
الاقتصادية في أي دولة.
في إطار "نموذج الانحدار الذاتيبنوزيع فترات الإبطاء" ،(Regressive Distributed Lag (ARDL Auto) و المعروف بإسم اختبار الحدود (Test Bounds). 3- فحص النموذج من خلال إجراء بعض الاختبارات

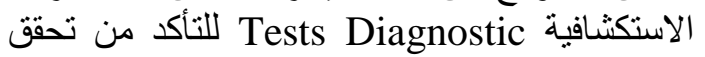

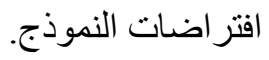
استخدام معدل نمو الناتج المحلي الإجمالي كمُتغير تابع يعبر عن النمو الاقتصادي واستخدام كلاً من نسبة الإدية

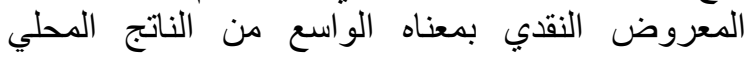

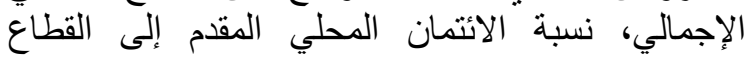

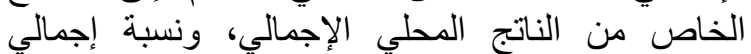

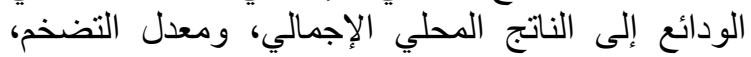

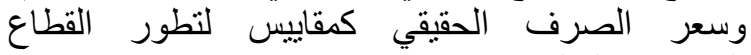

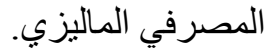

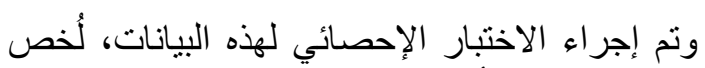

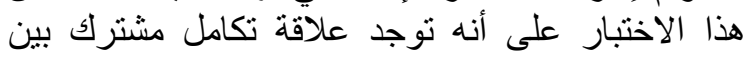

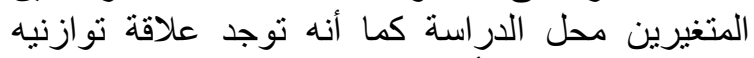

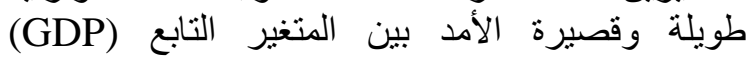
و المُتغير ات التفسيرية محل الأر اسة.

\section{النتائج والتوصبات}

\section{النتائج}

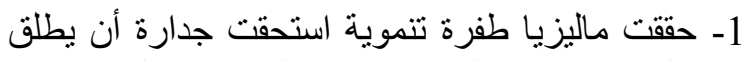

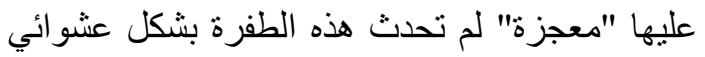
وغير مخطط؛ بل قامت الدولة الداء الماليزية ومؤسساتها بصياغة السياسات الاقتصادية المتعاقبة طويلة الأمد منذ عام 1971 إلى الآن بشكل تكاملت فيه هذه الأه الخطط.

2- ركزت ماليزيا على نموذج التنمية الياباني بشكل

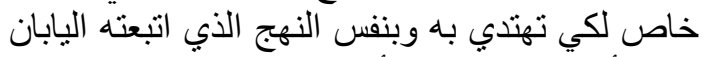

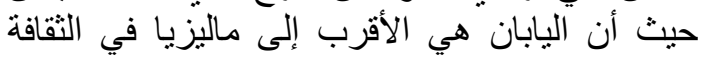
و الأخلاق ومحاو لة زيان زي الأرع هذه الثقافات لدى المآليزيين. 3- سياسة الخصخصة ليست الأمر السيئ كما يظن ولئن

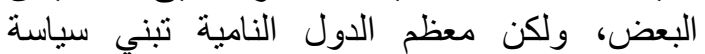
الخصخصة فيها على بيع أصول الدولة ولة والثركات الحكومية للأجانب، ولكن في الحئ ماليزيا نم بيعها للمو اطنين الماليزيين المخلصين لُوطنهم. 4- لم يكن النجاح الذي حققته التجربة التتموية الماليزية

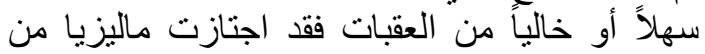

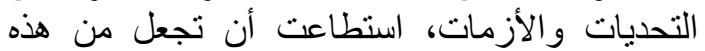

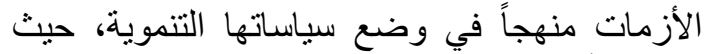

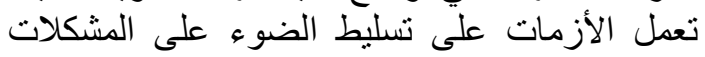

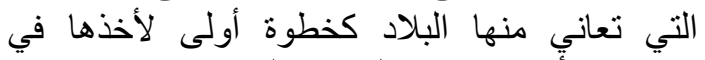
الاعتبار كأُحد مُدخلات السياسة العامة. 


$$
\text { المراجع }
$$

اندراوس، عاطف وليم (2008). أسواق الأوراق المالية

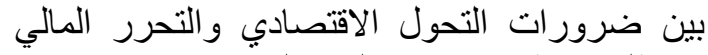
ومنطلبات تطوير ها، دار الفكر الجامعي، الإسكندرية، الطبعة الأولي.

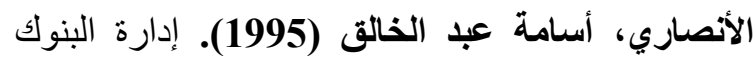

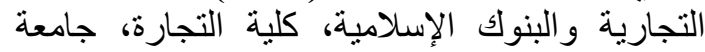

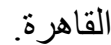

الجنابي، أرسلان (2009). رمزي ياسين، النقود و الهصارف و النظرية النقدية، دار و ائل للنشر ، الأردن.

الصباب، أحمد (2004). التخطبط و التنمية الاقتصادية في المملكة العربية السعودية، مركز النيطية النشر العلمي، جامعة الملك عبد العزيز.

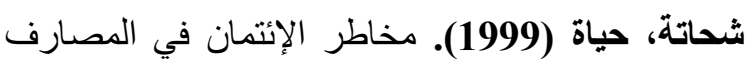

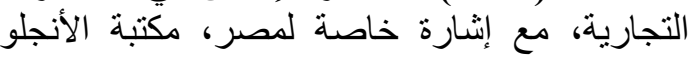

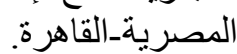

علي، أحمد شعبان محمد (2007). انعكاسات المتغيرات

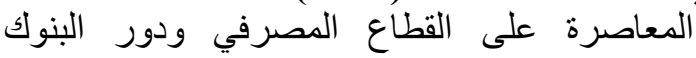

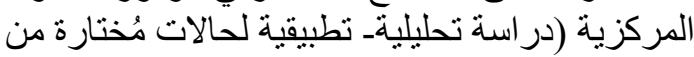
البلدان العربية)، الدار الجامعية، القاهرة.

Abdul Kareem, A.M.A. (1988). Korean economic Development Dependency approval, Unpublished Paper.

Chung, C.; Yang, D. and Joo, S. (2000). Foreign exchange market liberalization: The Case of Korea. Korea: Korea Inst. Int. Econ. Policy.

Terrick, B. and Kindlebebarger, C.P. (1985). Economic Development, London: McGraw-Hill Book Company.
5- يجب عدم نبذ الخصخصة للقطاع المصرفي ولكن

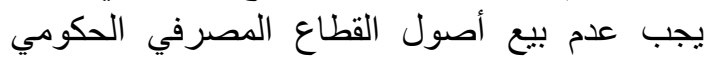
للأجانب بل يجب بيعه لرجال الأعمال الوطنيين داخل الفركي

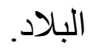
6- يجب أن يعمل القطاع المصرفي بشكل متناغم مع خطط التتمية الاقتصادية بحيث يتم دفع عجلة التنمية الاقتصادية.

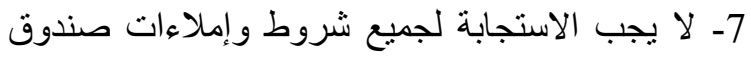

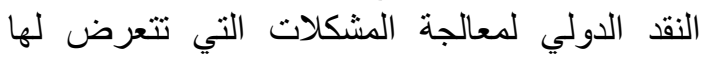

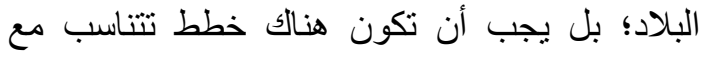

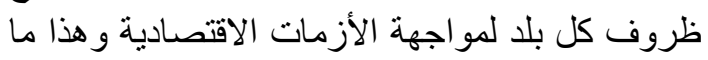
حدث في ماليزيا عندما قامت بمعالجة أزمنها بنفسها مما جعلها أسرع الدول خروجاً من الأزمة. 8- يجب أن تهنم الدول بالنظام المصرفي الإسلامي على النى

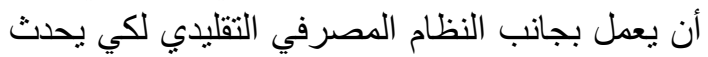

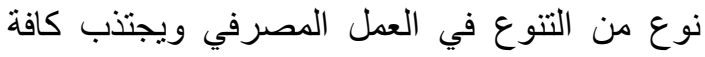

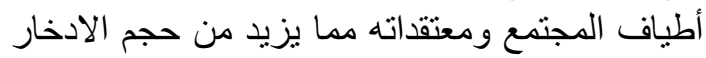
الموجه للاستثمار و التنمية.

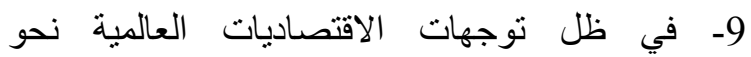

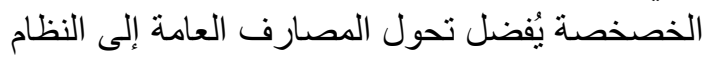

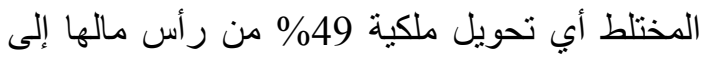

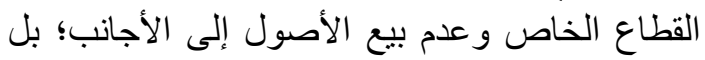
يتم طرح جزء من المصارف عن طريق سُوق المال مع جعل نسبة السبطرة للحكومة. 10- تقديم قروض متوسطة وطويلة الأجل بأسعار فائدة

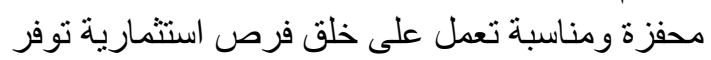

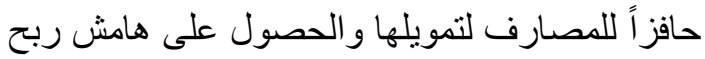
مناسب. 


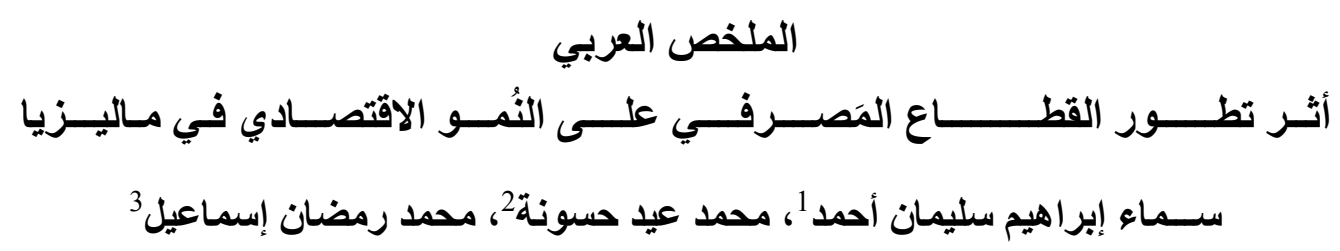

1- قسم در اسات وبحوث العلوم السياسية والاقتصادية، معهد الدر اسات و البحوث الآسيوية، جامعة الزقازيق، مصر.

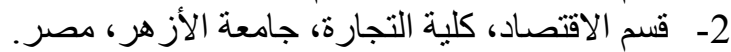

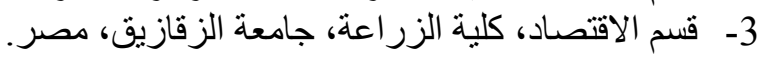

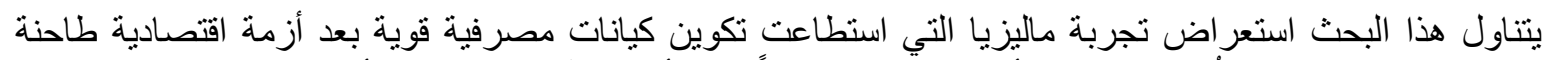

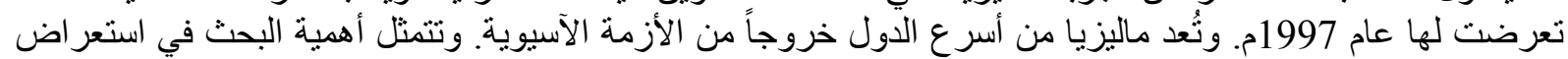

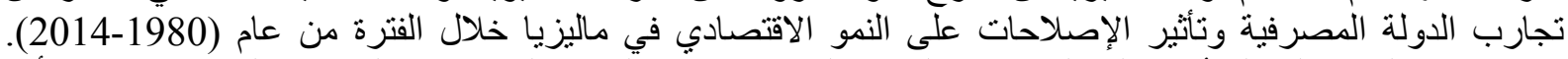

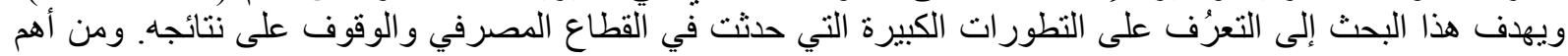

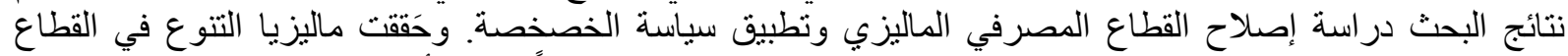

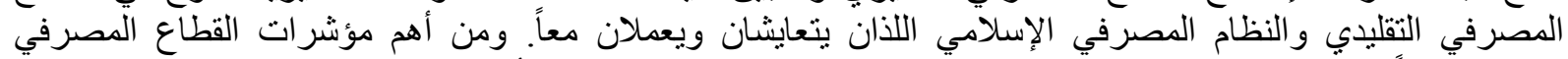

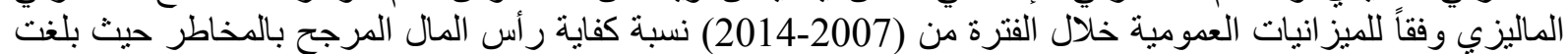

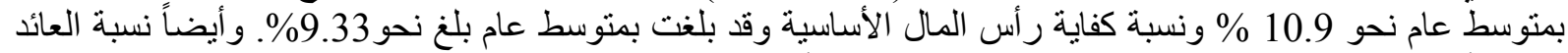

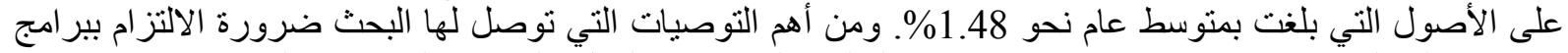

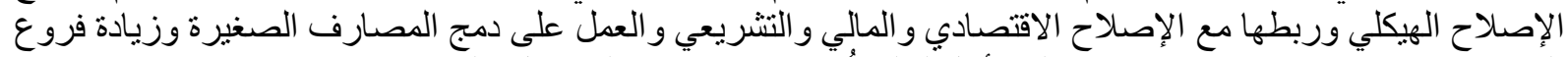

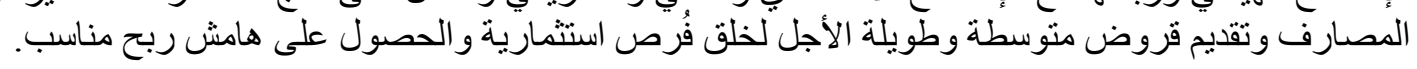

الكلمات الاسترشادية: القطاع المصرفي، الخصخصة، النمُو الاقتصادي، ماليزيا، بنك البومبيونر ا. 
